\title{
A battery-free non-intrusive power meter for low-cost energy monitoring
}

\author{
Giorgia Dalpiaz*, Alessandro Longo*, Matteo Nardello ${ }^{\dagger}$, Roberto Passerone ${ }^{\ddagger}$ and Davide Brunelli ${ }^{\dagger}$ \\ *Dept. of Information Engineering and Computer Science, \{name.surname\}@studenti.unitn.it \\ $\ddagger$ Dept. of Information Engineering and Computer Science, \{name.surname\}@unitn.it \\ ${ }^{\dagger}$ Dept. of Industrial Engineering, \{name.surname $\} @$ unitn.it \\ University of Trento, Trento, Italy
}

\begin{abstract}
As highlighted by the Intergovernmental Panel for Climate Change (IPCC), providing clean, reliable and affordable energy for people everywhere will require the reduction of gas emission in the energy domain by $90 \%$, compared to 2010 emissions, between the years 2040 and 2070. In addition, to a change in choices, preferences and behavior of individuals and households in energy demand and consumption, to achieve a successful transition to a more sustainable energy system, the adoption of new metering solutions to foster a wide range of sustainable actions by diverse people across the globe will be required. In this paper, we discuss the development of an ultra-low-power energy meter exploiting a single current transformer (CT) sensor for harvesting energy from the same load under monitoring. Starting from the hypothesis that the node activation rate increases monotonically with the primary load draw, and assuming that the node consumes fixed energy quanta during each activation, it is possible to infer the load power from the interval between activations. With this approach, we can provide a device that lowers maintenance cost related to installation and battery replacement; does not need to deal with high main voltage; and does not introduce any additional energy consumption overhead as it draws zero-power under zero-load condition. Energy budget is guaranteed also thanks to the use of a LoRa radio for data transmission.
\end{abstract}

\section{INTRODUCTION}

Since many people remain unaware of the links between the monthly energy expenditure and the power consumption of individual industrial machines or household appliances, it is useful to provide information about the way energy is used [1], [2]. The introduction of new smart metering devices offers a number of benefits for both the end user and utility companies. For instance, users can better understand which device is most energy hungry, thus take action to lower the associated energy expenditure, while utilities can profile the quantity and the quality of the energy delivered by power plants allowing them to take decisions that can avoid power outages and distribution losses. The low-cost of wireless sensor networks, combined with their ease of installation and flexibility, makes them the most attractive technology to implement a number of applications for smart metering, but the existing devices are usually expensive, require high installation cost due to physical intrusiveness and the need to tap into mains voltage for powering the device and, in the case of battery powered devices, they introduce maintenance costs related to battery replacement. Moreover, an additional energy overhead is added due to the power meter standby power, also when the load under measurement is switched off.

Several methods have been devised to harvest power from the environment to supply a sensor node, such as heat [3], vibrations [4] or sunlight [5]. In this paper we discuss the design, development and characterization of a power-proportional, energy-harvesting non-intrusive energy meter, that, thanks to a clamp-on Current Transformer (CT), is able to harvest the energy needed for its operation directly from the same load under monitoring. The proposed solution is equipped with an ultra-lowpower STM32L4 microcontroller from STMicroelectronics, that is in charge of configuring the radio, a LoRaWAN solution, that permit us to meet the energy budget for sending a packet also at low primary load, while still ensuring a transmission in the range of kilometers.

The working principle is simple: once the sensor is clamped around one phase of the main line, it starts to harvest energy from the load under monitoring. This energy is then stored inside an energy reservoir such as supercapacitors or lithium-ion capacitors [6]. In our case, we use a supercapacitor and when sufficient energy has been harvested, a sensor node powered by the supercapacitor turns on and sends a data packet to a remote server. Under steady-state conditions, we may assume that the power-on plus the packet transmission task consume a fixed amount of energy from the super capacitor. Therefore, because the rate at which the super capacitor is charged depends on the current drawn by the load, the node activation rate and consequently the packet transmission rate will also depend on the load power. After characterizing this dependency, the server can therefore estimate the load power simply from the rate at which it receives the packets.

This design offers several advantages over more traditional solutions. First, installation costs are lowered due to the nonintrusive approach that does not require to deal with primary main voltage, since there is no physical connection with the monitored circuit. Second, maintenance costs associated to battery replacement are avoided and the energy overhead introduced by the metering infrastructure is negligible and proportional to the load under monitoring, ensuring zero-power under zero-load condition. 


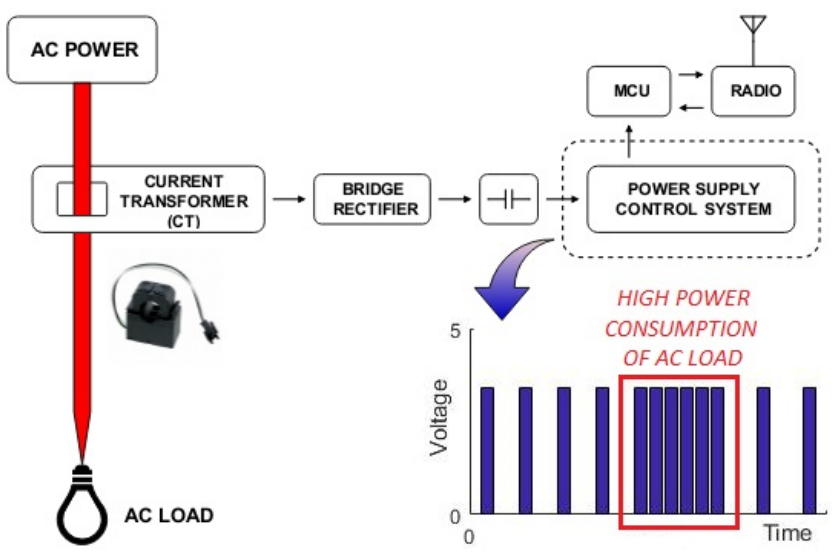

Fig. 1. Block diagram of the node architecture.

The rest of this paper is organized as follows: Section II present a brief account of the current state of the art in this area. Section III describe the system architecture and presents the motivations behind our architecture. Section IV discusses the hardware implementation, while results are discussed in Section V. Closing this work, Section VI discusses future improvements and draws final conclusions.

\section{RELATED WORK}

The constantly growing demand for energy efficiency driven by the targets established by the European Energy Efficiency Directive (EED) 2012/27/EU has stimulated the development of advanced systems for energy management and monitoring [7]. However, the development of an advanced metering infrastructure is still slow, because of invasiveness, scalability and cost issues. Next generation smart meters [8], [9] must feature a small form factor, have to be non-invasive, and must be reliable in terms of production and maintenance, considering the large number of expected measuring points. In order to meet this growing interest in solutions to better track energy consumption, both industrial and academic research have designed different metering solution, from the most basic plug-load meters, to circuit/panel meters capable of detecting different loads starting from the aggregate consumption.

Plug-load meters [10]-[14], are the first introduced solution for monitoring energy consumption. They are usually accurate and reliable in monitoring individual loads, but the drawbacks introduced are usually more than the advantages. First of all, they are active devices, meaning that they draw current also when the load to be monitored is switched off, increasing the metering overhead. While individually small, this amount can grow to be non-negligible in the case of more than a few measurement points. Another common issue is that sometimes attaching a plug load meter is impossible, for instance in the case of loads that are hard to move, like lighting systems or kitchen appliances.

To address some of the problem of this type of metering devices, researchers have proposed the use of circuit level meters [15]-[17], but they usually represent a barrier for most of the users, as they can be difficult to install and to retrofit in existing infrastructure. To overcome high installation costs that can limit the widespread adoption of a metering infrastructure, recent meters are exploring various non-contact options that, combined with energy harvesting power supply, can represent a feasible solution to achieve a distributed power monitoring infrastructure. Nke-watteco [18] has recently started to offer a battery powered LoRaWAN power meter, with an expected battery life of 10 years, while, Patel et al. [19] designed a stick-on power meter using magnetometers designed to be attached to the circuit breaker. Although good solutions, both devices rely on a battery as their power source, representing a problem if deployed in scale.

Energy-Harvesting power meters obviate the problem of battery recharge and replacement. For example, DeBruin et al. present a non-intrusive wireless current monitoring system that integrates an energy harvesting power supply [13]. Power consumption is then inferred by the node activation frequency that increases monotonically with the primary load draw. Our design differs in the range of load power levels and transmission technology. Porcarelli et al. [9] propose a system that exploits a single CT sensor, used for both energy harvesting and current sensing. The node is configured to sense current while in active mode and to harvest energy when in sleep mode.

\section{SYSTEM OVERVIEW}

Our design choices have been driven primarily by three properties that characterize the system: non-intrusiveness, batteryfree operation and low power consumption. In accordance with these properties, we have employed a clamp-on inductive 
current sensor which functions as the transformer. This type of transformer allows the device to be non-intrusive, since it can be deployed easily without modifying the circuit and the device under measurement. In addition, the transformer is the sole energy supplier of the node, which operates without the use of any battery. The meter can therefore operate completely unattended. Because there is no actual sensing device with its associated sampling and conversion circuit, the node architecture is extremely simple. The direct consequence is the implementation of an extremely low power transmission system, an essential feature that increases the effectiveness of the harvesting solution.

According to the implemented choices, we can monitor the load consumption by analyzing the rate of the transmitted packets. This is possible thanks to the correlation between the charging speed of the capacitor and the current absorbed by the primary load. This technique for monitoring electricity consumption has these advantages:

- No use of peripheral devices is required by the MCU to perform the measurements. This includes the current transformer, which is used as a harvester and only acts indirectly as a current sensor. This approach drastically decreases the overall power consumed by the node, which is limited to setting up the radio and sending a packet, without any kind of data processing, simplifying the energy harvesting task.

- The MCU remains deactivated for the entire capacitor charging period, and is activated only to carry out a transmission. Nevertheless, because the harvester continuously integrates the generated current into the capacitor, the node is effectively measuring the power consumption even during the sleep periods of the MCU. In other words, we do not have to deal with problems related to infrequent sampling or lost data, nor do we need to worry about changes in the load power level, since these are automatically accounted for. The method is therefore suitable to monitor individual appliances, as well as the main power distribution lines.

- The packet does not need to contain any measured value, since power consumption is inferred by the frequency of the transmissions. It is therefore possible to decrease the number of bits sent for every transmission, thus reducing transmission time and consumption. This is especially relevant in the context of long range transmissions, which are unable to carry large amounts of data. Conversely, data packets could be delivered more infrequently by collecting the wake-up times in the local memory, and then transmitting the information only once every $n$ activations. By doing so we can strike the best trade-off between node power consumption and data availability, without sacrificing accuracy.

Because we estimate the energy consumption from the packet transmission rate, we also have to correctly receive all the packets. If packets get lost during transmission, the energy consumption will be underestimated leading to an error. To mitigate this problem, the meter inserts in the payload of the packet a monotonically increasing counter along with the timestamp of the last 3 activations. The server can therefore recover lost packets with very high probability.

\section{ENERGY METER HARDWARE DESIGN}

The block diagram in Figure 1 shows the overall architecture and working principle of the proposed system. A current transformer (CT) is clamped around one phase of the supply line going from the mains to the load that we intend to monitor. The supply line acts as the primary winding of the CT. As the load draws power from the mains, a current proportional to the load consumption is induced in the secondary winding of the CT and can be harvested by storing it in an energy reservoir, in our case, a super-capacitor. To harvest this energy, the current must be rectified through a diode bridge before it is stored in the super-capacitor. A microcontroller (MCU) and a radio transmitter are connected to the capacitor through a power supply control system and are normally switched off, to eliminate energy waste when the super-capacitor is recharging. When the voltage across the capacitor reaches a predetermined threshold, the control system activates the microcontroller, which in turn starts a wireless transmission, which is then captured by a remote server. At the end of the transmission, the microcontroller and the radio are deactivated. The operation draws energy from the capacitor, whose voltage decreases back below the threshold. At the same time, the harvester starts to recharge the capacitor, at a rate that is proportional to the power drawn by the load from the main line. Thus, once the voltage crosses the threshold again, a new transmission operation is started by the MCU. This cycle repeats at a rate proportional to the energy harvested from the load: the higher the load power level, the more frequent the packet transmission, as shown in the inset of Figure 1. Hence, the packet transmission rate depends on the load power, which can therefore be estimated by the remote server by simply counting the number of received packets per minute. In other words, the transformer supplies the entire circuit and the whole system acts at the same time as a current sensor.

In the rest of this section we provide more details on the individual components that constitute our architecture.

\section{A. Current Sensor}

The current sensor consists of a clamp-on inductive sensor, applied to the load. For harvesting the energy needed to power the board, as previously stated, we have used a Current Transformer (CT), that is clamped around one of the phases that goes to the load that we want to monitor. Different tests were conducted to determine the most suitable sensor for this kind of application. Two different sensors have been compared. A LEM TOP90-S10 / SP2 with a 1:1000 ratio and a VITEC 57PR1673 with a 1:3000 ratio. Contrary to what we would have expected, the sensor with 1:3000 ratio, that theoretically should provide one third of the current compared to the former, performs much better. Thus, experimental tests were conducted with the latter. 


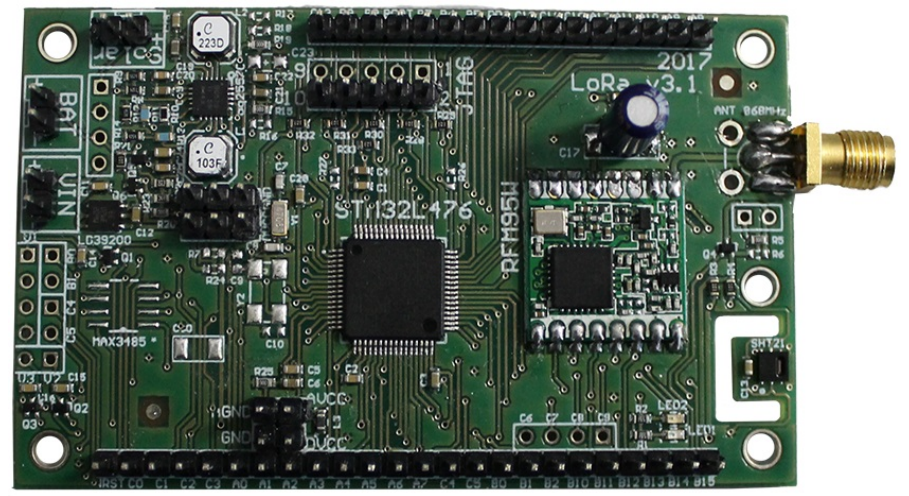

Fig. 2. Assembled board with microcontroller and radio.

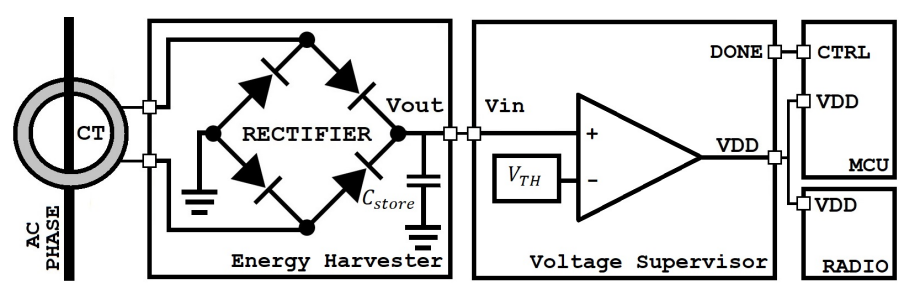

Fig. 3. Power supply control system block schematics.

\section{B. Rectifier circuit}

The current rectifier is of fundamental importance, since it affects the overall efficiency of the energy harvester. Our circuit consists of a Graetz bridge with Schottky diodes, featuring a low forward active voltage. To choose the right diode for this type of application, our research has started by searching for the diode with the lowest forward voltage and leakage current. Once a range of diodes was selected, tests were performed to determine the one with the best efficiency at lower loads. After some experiments, we have chosen to use four STMicroelectronics BAT30 diodes, as they proved to be the most efficient at lower load level, even though not the most efficient at higher loads. The motivation behind this choice is that we are more interested in higher efficiency when the energy that can be scavenged is limited, in order to increase the node activation rate at lower primary loads. Conversely, at higher primary loads we have plenty of energy for powering the meter, so the lower efficiency of the selected diode can be neglected.

\section{Capacitor}

The capacitor is the heart of the system as it is in charge of storing the energy harvested and needs to be properly sized. It must guarantee sufficient energy for the microcontroller operation and for the radio to transmit a packet, but also not represent a barrier for the activation at lower loads. After some experimental tests and measurements, the AVX Best Cap $22 \mathrm{mF} / 4.5 \mathrm{~V}$ supercapacitor was chosen as the best compromise between charge times and stored energy.

\section{Power supply control system}

The power supply control system activates/deactivates the MCU, allowing or preventing the flow of current from the capacitor. At the current state of work, this component is still a prototype, as its operation must be carefully calibrated according to the harvester and the node characterization. In the tests that we have carried out, a completely autonomous power supply system was used, so that no external factor would influence our measurements. It is mainly composed by an ultralow power Voltage Supervisor, that activates its output only when the input reaches a fixed threshold. Moreover, it provides a "done" input pin that can be used to disable the output. This pin is connected to the MCU that is configured to send a signal when the packet has been sent. In this way, we keep the MCU and radio totally disconnected while the super capacitor is recharging. The power supply is shown schematically in Figure 3.

\section{E. Microcontroller and radio}

The microcontroller is a low-power STM32L4 from STMicroelectronics, chosen for its versatility and reduced energy consumption, while the radio used for transmission is the RFM95W, based on a LoRa modem, which provides ultra-long-range transmission and high interference immunity while still meeting the energy budget of the harvesting power supply. The board, 


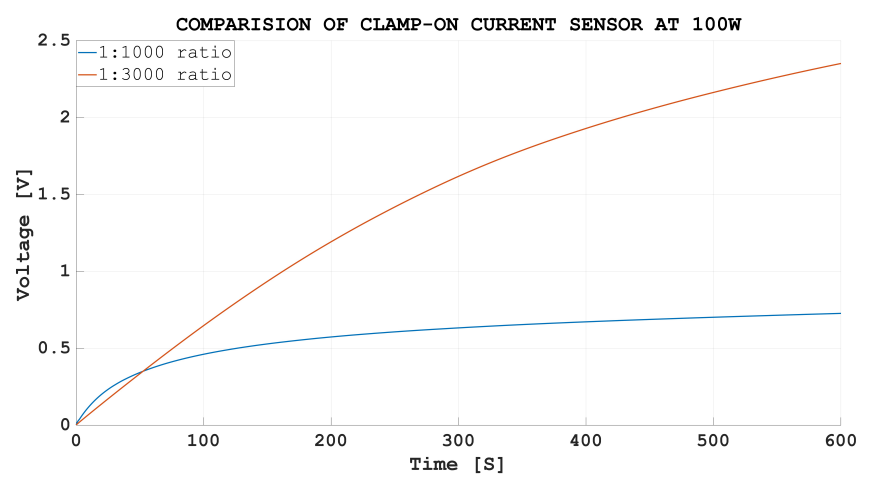

Fig. 4. Super capacitor voltage trend using different transformer ration. Contrary to the expected behavior, the CT sensor with a higher number of turns better match the energy harvesting system, leading to a higher efficiency in charging the super-capacitor.

assembled with all its components is shown in Figure 2. This is a first prototype, that integrates a high power LoRa radio, that does not permit the use of low power amplifier. We have used this platform, as we wanted to characterize the worst case scenario. Future work will use another LoRa radio chip, that permits us to fully exploit the various transmission power levels. Moreover, to further reduce the power requirement, a smaller and less powerful microcontroller could be used.

\section{EXPERIMENTAL RESULTS}

As our main goal was the full characterization of the relation between the transmission rate and the load power level, our experiments have evaluated different device solutions for the node architecture, and the characterization of both the energy required for data transmission and the rate at which energy can be harvested and stored in the super capacitor. The experimental data are essential in order to correctly calibrate the measurement system, since we need to quantify the energy quanta needed for transmitting one packet and then link this parameter to the load power consumption to express a relation between the load level and the activation rate.

A first set of experiments was intended to characterize the performance of two current transformers, having respectively a 1:1000 and 1:3000 ratio. Because in a CT there is an inverse relationship between the number of turns in the secondary windings and the induced current, we expect the transformer with the lower number of turns to perform better, i.e., to charge the super-capacitor more quickly. However, other parameters, such as the impedance mismatch with the non-linear rectifying circuit, which ultimately determines the amount of energy that can effectively be transferred, and the geometric shape of the sensor, must also be taken into account. Various measurements were performed, showing how these secondary effects have a significant impact on the overall performance, leading to the use of the sensor with a higher ratio. The result of this analysis is presented in Figure 4. While the 1:1000 sensor behaves better at the very beginning of the charging cycle, the overall charging time at $100 \mathrm{~W}$ with the 1:3000 sensor is considerably lower, denoting a better efficiency compared with the other one.

Besides the current sensor, a set of experiments has also been conducted to test different diode bridges to select the one that is more suitable for this kind of application. We have decided to use the one with a higher efficiency at the lower load levels, even if not the best at higher load levels. This is because we are more interested in the best measurement granularity at lower loads, as the energy that can be scavenged at higher loads is always sufficient to meet the energy budget of the platform. This analysis has lead us to use a BAT30 diode. Figure 6 shows the result of these experiments, where the charge level of the super capacitor over time is compared among the different diode bridges for a $500 \mathrm{~W}$ load. The differences are due to the different forward resistance of the devices as a function of the current level. To simulate the various main loads, a variable load made by a cluster of incandescent light bulbs has been used. This cluster permits us to vary the load from 50 to 1600 watt in $50 \mathrm{~W}$ steps.

Once the issues related to the current sensor and the most efficient bridge rectifier had been addressed, it was necessary to size the capacitor. For this purpose, we have collected the current consumption curve of the node platform during one operation cycle (i.e., Power on, configuration and packet transmission), and then calculated the energy required for the task, which is equal to $14.5 \mathrm{~mJ}$ at $+20 \mathrm{dBm}$. As the energy is drawn from the capacitor, the node supply voltage decreases. For correct operation, the voltage should not decrease below the radio operating limit, which we fix at $3 \mathrm{~V}$. We can therefore compute the value of the capacitor, by considering the energy stored at the desired voltage levels. We obtain a capacitance of approximately $11 \mathrm{mF}$. Given the device tolerance, and to provide sufficient operating margins, we have employed a $22 \mathrm{mF}$ capacitor, because it stores enough energy to supply both the microcontroller and the radio during the transmission, guaranteeing however reasonable charge times. 


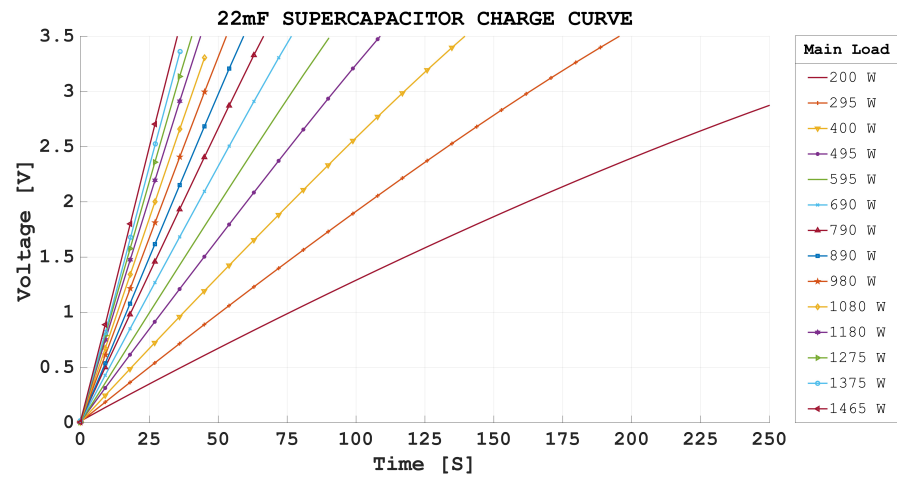

Fig. 5. Voltage trend of the $22 \mathrm{mf}$ super-capacitor while harvesting energy from different primary loads.

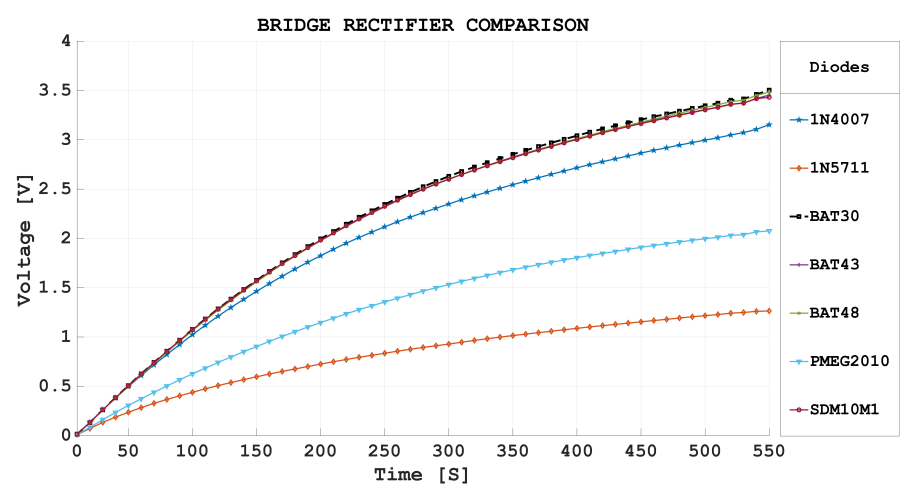

Fig. 6. Super-capacitor charge profile for a $130 \mathrm{~W}$ load using different rectifier bridges. The dashed line (BAT30), represent the chosen solution. We chose this diode as more efficient at lower load, in order to extend the functionalities of the meter also at lower primary loads.

Figure 5 shows the charging profile of the capacitor over time as the load varies from $200 \mathrm{~W}$ to $1465 \mathrm{~W}$. As the energy needed to ensure a failsafe LoRa transmission is met at $3.39 \mathrm{~V}$, the graphs show the charging time to reach that threshold. Naturally, the lower the load the longer the time required to reach the threshold. It can be noted that the platform exhibits a reasonable charging time starting from $200 \mathrm{~W}$.

Figure 7 shows the curve obtained by interpolating the measured full charging time at different load power levels, starting from a fully discharged capacitor. In other words, it shows the minimum load power required to reach the voltage threshold given a required time. The estimated curve represents a branch of an equilateral hyperbola. In contrast to the fitting curve, the measurements (the dots in the figure) show that the lower power loads need slightly more time to reach the threshold voltage compared to the estimated time. This is due to the non-linear behavior of the circuit, with the non-ideal parameters having a more pronounced effect at lower loads and currents.

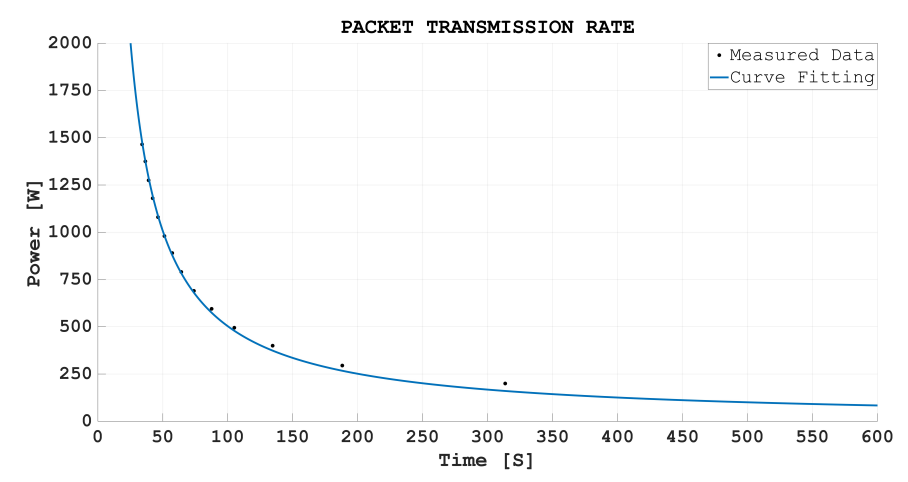

Fig. 7. Packet transmission rate at different primary loads. 

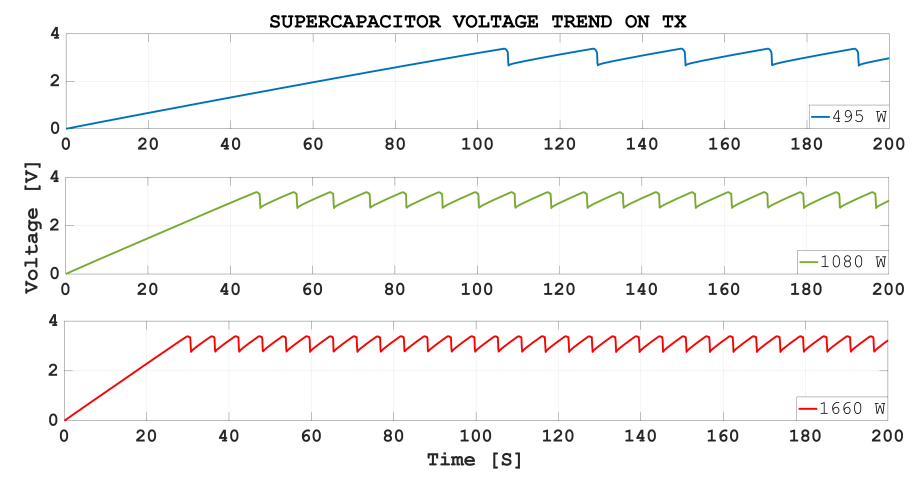

Fig. 8. Super-capacitor voltage drop due to a packet transmission at different main loads. The amount of energy for transmitting a packet remains constant through different loads, while the transmission rate increases as the primary load increases.

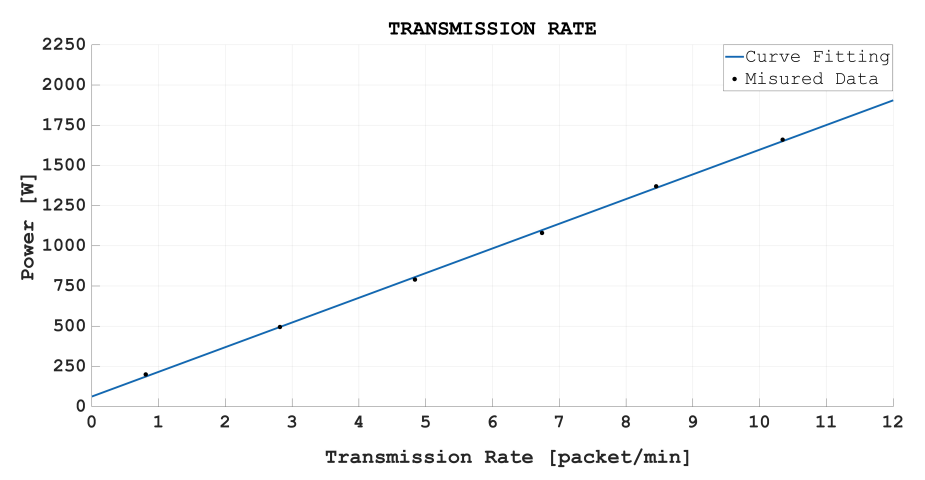

Fig. 9. Relation between main load and Packet rate [packet/min].

In the next step of the experimentation, we have analyzed the behavior of the capacitor during the transmission period. The radio has been programmed to transmit a packet with a reduced number of bits. In these measurements, the packet is transmitted with a maximum gain, equal to $+20 \mathrm{dBm}$ and a spread factor of 12 , to simulate the worst working condition. Of course, in case we do not require the maximum transmission range, these parameters can be relaxed, leading to a lower energy requirement, meaning a higher activation rate and accuracy. At the end of each transmission, the microcontroller sends a signal to the power supply control system, which in turn inhibits the current flow, so that the capacitor can start to charge again. Figure 8 shows the trend of the voltage across the capacitor during the initial and the full operation period, for three different primary load power levels. As expected, the packet transmission rate depends on the load and remains constant if there are no power variations.

On the basis of the data shown above, it is easy to relate the load power level to the steady state packet transmission rate. This relation is shown, together with its linear interpolation, in Figure 9. In contrast to the initial full charge time, the relation is close to linear, since the capacitor is only partially discharged and the energy required to bring its voltage back to the threshold is much smaller than that required during start up. We observe that even at low power levels, we are still able to send one packet per minute. This confirms that the system can operate on a wide range of loads.

\section{CONCLUSION}

We have presented a battery-free, non-intrusive power meter for low-cost energy monitoring. The proposed system works and respects the three properties initially imposed. The characterization is used to determine the relation between the packet transmission rate and the measured load power level. Thanks to its versatility, the device could be used for a wide range of applications. For example, the long-distance transmission of the radio permits to supervise remote devices, without battery support. The characterization shows that the optimal operation is obtained with medium-high power loads, with rates of several packets per minute. At the same time, lower power levels are still monitored and duly reported to the server, despite the lower packet transmission rate, as the node continuously integrates the current from the supply line. In addition, the radio gain could be considerably reduced, whenever the collecting server is close to the sensor. This would decrease significantly the energy that needs to be stored on the capacitor, leading to much faster charging times. 
Our future work includes optimizing the system when it works with a low power consumption load. This will guarantee a complete range of measurement on commonly used devices, including more recent lower power lighting and battery chargers. In particular, it is possible to change the capacitor in order to obtain devices with different measuring times and various resolutions, by adopting different data transmission strategies. Finally, it is necessary to complete the design of the power supply control system unit and calibrate the operation, considering the extra consumption introduced in the system.

\section{REFERENCES}

[1] D. Brunelli et al., "Smart monitoring for sustainable and energy-efficient buildings: A case study," in IEEE Workshop on Environmental, Energy, and Structural Monitoring Systems (EESMS), July 2015, pp. 186-191.

[2] D. Brunelli et al., "POVOMON: an ad-hoc wireless sensor network for indoor environmental monitoring," in IEEE Workshop on Environmental, Energy and Structural Monitoring Systems (EESMS), September 2014.

[3] M. Rossi et al., "Energy neutral wireless sensing for server farms monitoring," IEEE J. on Emerging and Selected Topics in Circuits and Systems, vol. 4, no. 3, pp. 324-334, Sept 2014.

[4] I. Minakov et al., "Design and energy optimization of a multifunctional iot solution for connected bikes," in Global Internet of Things Summit (GIoTS), June 2017, pp. 1-6.

[5] C. Bergonzini et al., "Comparison of energy intake prediction algorithms for systems powered by photovoltaic harvesters," Microelectron. J., vol. 41, no. 11 , pp. 766-777, Nov. 2010.

[6] D. Porcarelli et al., "Characterization of lithium-ion capacitors for low-power energy neutral wireless sensor networks," in Ninth International Conference on Networked Sensing (INSS), June 2012, pp. 1-4.

[7] D. Brunelli et al., "Smart grid configuration tool for hees systems in smart city districts," in Int. Symposium on Power Electronics, Electrical Drives, Automation and Motion (SPEEDAM), June 2016, pp. 137-142.

[8] S. Makonin et al., "Inspiring energy conservation through open source metering hardware and embedded real-time load disaggregation," in $I E E E$ PES Asia-Pacific Power and Energy Engineering Conference (APPEEC), Dec 2013, pp. 1-6.

[9] D. Porcarelli et al., "Clamp-and-forget: A self-sustainable non-invasive wireless sensor node for smart metering applications," Microelectron. J., vol. 45, no. 12 , pp. 1671 - 1678, 2014

[10] Belkin WeMo Insight. http://www.belkin.com/us/p/P-F7C029/

[11] S. DeBruin et al., "Demo: Powerblade a low-profile, true-power, plug-through energy meter," in 13th ACM Conference on Embedded Networked Sensor Systems, ser. SenSys '15, 2015, pp. 463-464.

[12] M. Nardello et al., "A low-cost smart sensor for non intrusive load monitoring applications," in IEEE 26th International Symposium on Industrial Electronics (ISIE), June 2017, pp. 1362-1368.

[13] S. DeBruin et al., "Monjolo: An energy-harvesting energy meter architecture," in 11th ACM Conference on Embedded Networked Sensor Systems, ser. SenSys '13, 2013, pp. 18:1-18:14

[14] Kill A Watt EZ. http://www.p3international.com/products/p4460.html

[15] TED: The Energy Detective. http://www.theenergydetective.com

[16] N. Klingensmith et al., "A distributed energy monitoring and analytics platform and its use cases," in 5th ACM Workshop on Embedded Systems For Energy-Efficient Buildings, ser. BuildSys'13, 2013, pp. 5:1-5:8.

[17] eGauge Pro meter. http://www.egauge.net/eos/energymeters/EG4130

[18] IntensO Sensor. http://www.nke-watteco.com/product/intensity-sensor-lorawan

[19] S. N. Patel et al., "The design and evaluation of an end-user-deployable, whole house, contactless power consumption sensor," in SIGCHI Conf. on Human Factors in Comp. Sys., ser. CHI' 10, 2010, pp. 2471-2480. 